\title{
Signal Exclusive Adaptive Average Filter for Impulse Noise Suppression
}

\author{
Yunhua Wang, Linda S. DeBrunner, Joseph P. Havlicek, and Dayong Zhou \\ School of Electrical \& Computer Engineering \\ University of Oklahoma, Norman, OK 73019 USA \\ \{xiao9, ldebrunner, joebob, dayong\}@ou.edu
}

\begin{abstract}
This paper introduces a novel signal exclusive adaptive average (SEAA) filter that offers good image denoising performance in applications characterized by impulsive or impulse-like noise. The proposed algorithm works well in suppressing impulse noise with noise ratios from $3 \%$ up to $60 \%$. We begin by introducing a digital differentiation preprocessing step to quantify the increments in each local neighborhood of the noisy image. A homogeneity level map is then derived by adaptive thresholding and used to designate pixels as noise candidates. The initial selection is refined using a novel connected components labeling algorithm. Finally, the noise is attenuated by estimating the values of the noisy pixels with a linear filter applied exclusively to those neighborhood pixels not labeled as noise candidates. This approach bears similarity to several nonlinear techniques including alpha-trimmed means, selective averaging, and WMMR filters. Simulation results indicate that SEAA is better able to preserve 2-D edge structures from the original image and delivers better performance with less computational overhead as compared to competing nonlinear denoising algorithms.
\end{abstract}

\section{Introduction}

In acquisition and transmission applications, images are frequently corrupted by impulse-like noise arising from, e.g., faulty sensors, "stuck-at" bit errors, quasi-stationary channel defects, and other nonlinear effects [1]. Noise models of this type can be well described as follows. Let $I(i, j)$ be a pixel from the original gray scale image $I$ that is located at position coordinates $(i, j)$, where $I(i, j)$ takes values in the range $\left[I_{\min }, I_{\max }\right]$. Let $J(i, j)$ be the noise corrupted image. We consider that

$$
J(i, j)= \begin{cases}N(i, j) & \text { with prob. } p, \\ I(i, j) & \text { with prob. } 1-p,\end{cases}
$$

where $N(i, j) \in\left[I_{\text {min }}, I_{\text {max }}\right]$ are random deviates with noise ratio $p$ and an underlying distribution that is assumed known. One well-known special case is when $N(i, j)$ takes the values $I_{\text {min }}$ and $I_{\text {max }}$ with equal probability; this is called fixed-value impulse noise or salt and pepper noise [2]. More generally, noise processes of this type are referred to as random impulse noise, where the values of $N(i, j)$ are often uniformly distributed in the range $\left[I_{\min }, I_{\max }\right]$.

The most popular approaches for dealing with such noise have been based on median filtering and/or on the rich class of order statistic filters that have emerged from the study of median filters [2]. Recently, variations on the median filtering theme have been shown, under various specific signal/noise models, to deliver improved performance relative to the corresponding traditional methods. Examples include the exclusive mean filter (MMEM) [3], the adaptive center-weighted median filter (ACWMF) [4], and the median filter based on homogeneity information (CSAM) [5]. These filters have all demonstrated excellent performance, but at the price of significant computational complexity. The main drawback that characterizes all approaches of this type is that they involve computational overhead to determine one or more of the local order statistics. Moreover, while such filters are generally superior to linear filters for attenuating heavily tailed and impulse-like noise, order statistic filters and their relatives still tend to blur or degrade intrinsically 2-D edge structures such as corners that are reasonably expected to occur in typical images of the type found in most practical applications.

To ameliorate these problems, we propose in this paper a new algorithm that is computationally much simpler and that exhibits improved performance in removing impulse noise while preserving fine details 
of the 2-D image structure. This proposed approach works well not only for small noise ratios of about $3 \%$, but also for noise ratios as high as $60 \%$.

The signal exclusive adaptive average (SEAA) algorithm is presented in Section 2, while its effectiveness is studied by simulation in Section 3. In Section 4, we compare the SEAA algorithm with the recently proposed CSAM method. Conclusions appear in Section 5.

\section{The SEAA filter algorithm}

SEAA is a nonlinear adaptive filtering algorithm consisting of two major components: corrupted pixel detection and spatially localized noise filtering, which are implemented in five processing steps as shown below in Fig. 1 and described in the following paragraphs.

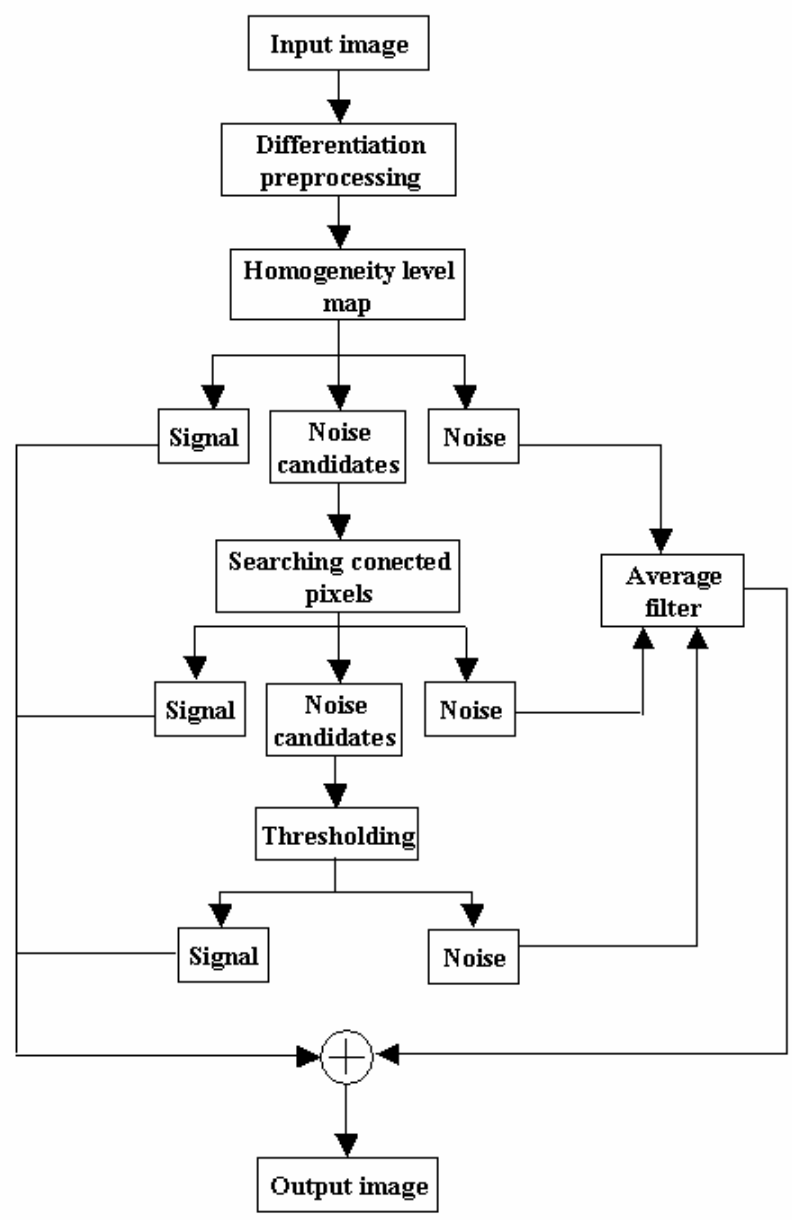

Figure 1. SEAA block diagram.
Step 1: Digital differentiation preprocessing. In order to obtain a quantitative characterization of the local variations in the observed noisy image $J$, we compute the local increments in a $3 \times 3$ window centered about each pixel $J(i, j)$, which may be thought of as a crude discrete approximation of the image gradient field. Define four linearly filtered difference images according to

$$
\begin{aligned}
& F J_{1}(i, j)=J(i+1, j)-J(i, j), \\
& F J_{2}(i, j)=J(i, j+1)-J(i, j), \\
& F J_{3}(i, j)=J(i+1, j+1)-J(i, j), \\
& F J_{4}(i, j)=J(i+1, j-1)-J(i, j) .
\end{aligned}
$$

The images $F J_{1}$ through $\mathrm{FJ}_{4}$ are used to construct the $3 \times 3$ window of 8-neighbor increments $W_{i, j}$ associated with the pixel $J(i, j)$ as shown in Fig. 2 below. By convention, we take $W_{i, j}(2,2)=J(i, j)$. If the image $J$ is being processed on the fly in raster scan order, it is worth noting that only the four increments $F J_{k}(i, j)$, $k=1, \ldots, 4$, need be calculated explicitly to construct $W_{i, j}$ : the other four increments in $W_{i, j}$ will already have been obtained during the construction of previous windows $W_{r, s}$ that are causal with respect to $W_{i, j}$ in the sense that $r<i$ or that $r=i$ and $s<j$.

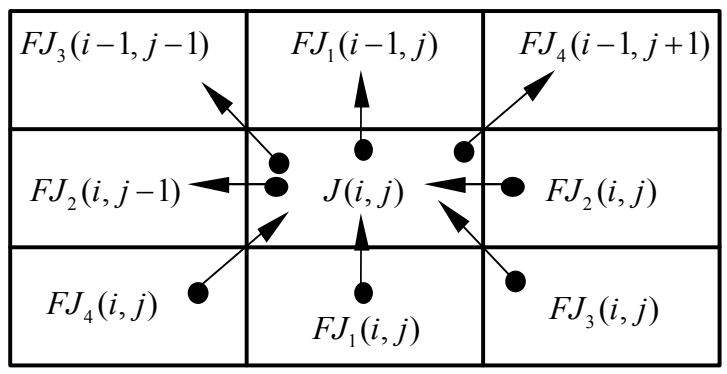

Figure 2. Window of increments $W_{i, j}(k, l)$, $1 \leq k, l \leq 3$, associated with the pixel $J(i, j)$.

Step 2: Calculation of homogeneity map by thresholding. In this step, we make use of the notion of homogeneity level from [5], which is defined based on the pairwise correlations between neighboring image pixels. We extend this notion to derive the $3 \times 3$ homogeneity level map $M_{i, j}$ associated with each pixel $J(i, j)$ by adaptively thresholding the window of increments $W_{i, j}$ according to 


$$
M_{i, j}(k, l)=\left\{\begin{array}{rc}
-1, & W_{i, j}(k, l)<-\alpha, \\
0, & -\alpha \leq W_{i, j}(k, l) \leq \alpha, \\
+1, & W_{i, j}(k, l)>\alpha,
\end{array}\right.
$$

where $\quad 1 \leq k, l \leq 3, \quad(k, l) \neq(2,2) . \quad$ Thus, $\quad$ e.g., $M_{i, j}(2,1)=0 \quad$ indicates a homogeneity or "connectedness" condition in the sense that the observed pixel value $J(i, j-1)$ supports the hypothesis that $J(i, j)$ is noise free - an event that, according to (1), occurs with probability $1-p$, whereas $M_{i, j}(2,1)= \pm 1$ indicates that the observed value $J(i, j-1)$ supports the hypothesis that $J(i, j)$ has been corrupted by noise. In (2), $\alpha$ is a nonlinear adaptive threshold that should be designed as a function of $J(i, j)$ based on the particular noise model.

In the case of eight-bit pixels corrupted by salt and pepper noise with $I_{\min }=0$ and $I_{\max }=255$, we take

$$
\alpha=\left\lceil 41-0.00234 \cdot[J(i, j)-127.5]^{2}\right\rceil .
$$

While empirical in nature, this approach is both simple and reasonable: unless supported by the occurrence of accompanying zero entries in $M_{i, j}(k, l)$, isolated instances of $J(i, j)=I_{\text {max }}, I_{\text {min }}$ are very likely to be caused by noise when the original image is corrupted by a salt and pepper noise model. From (3), it is readily seen that $\alpha$ is close to its minimum value when $J(i, j)$ is close to 0 or 255 and is larger otherwise.

Step 3: Noise and noise candidate detection. Initially, all pixels $J(i, j)$ are labeled as noise free. The homogeneity level maps $M_{i, j}$ are then processed to detect noise and noise candidate pixels. If the 8neighbors of $J(i, j)$ are all labeled with +1 or all labeled with -1 in $M_{i, j}$, then $J(i, j)$ is labeled as a noise pixel. Otherwise, if $M_{i, j}$ contains a mix of the labels $+1,-1$, and 0 , then it is possible that $J(i, j)$ is part of an edge structure in the image. We consider the hypothesis that $J(i, j)$ is noise free to be supported if the number of zero labels in $M_{i, j}$ is at least $\beta$, where we choose $3 \leq \beta \leq 7$ depending on the assumed value of $p$ in (1). Larger values $p$ generally require smaller choices for $\beta$. If $M_{i, j}$ contains a mix of labels and the number of zeros is less than $\beta$, then $J(i, j)$ is labeled as a noise candidate. Four examples $M_{i, j}$ leading to this designation are depicted in Fig. 3.

\begin{tabular}{|c|c|c|}
\hline-1 & -1 & +1 \\
\hline-1 & $J(i, j)$ & 0 \\
\hline-1 & +1 & -1 \\
\hline
\end{tabular}

\begin{tabular}{|c|c|c|}
\hline-1 & -1 & -1 \\
\hline 0 & $J(i, j)$ & 0 \\
\hline-1 & +1 & -1 \\
\hline
\end{tabular}

\begin{tabular}{|c|c|c|}
\hline-1 & -1 & 0 \\
\hline+1 & $J(i, j)$ & 0 \\
\hline+1 & +1 & -1 \\
\hline
\end{tabular}

\begin{tabular}{|c|c|c|}
\hline+1 & -1 & +1 \\
\hline 0 & $J(i, j)$ & 0 \\
\hline-1 & 0 & -1 \\
\hline
\end{tabular}

Figure 3. Examples of noise candidate maps $M_{i, j}$.

Step 4: Refinement of noise candidate selection. Each observed pixel $J(i, j)$ is connected to those 8neighbors for which the corresponding homogeneity map entry $M_{i, j}(k, l)$ is zero. Thus, the collection of maps $M_{i, j}$ defines connected components (sets of mutually connected pixels) within the observed image $J$. For each pixel labeled as a noise candidate, this notion of connected components is used to refine the label to noise or to noise free as follows. First, the set of pixels connected to $J(i, j)$ is searched for pixels that have already been assigned a label of noise or of noise free. The label of $J(i, j)$ is then changed to the label of the closest such pixel.

In case all of the pixels connected to $J(i, j)$ are noise candidates, a final label is assigned to $J(i, j)$ based on the size of this connected component. If the number of pixels in the component exceeds $\lambda$, then $J(i, j)$ is labeled as noise free. Otherwise, $J(i, j)$ is labeled as noise. The threshold $\lambda$ must be tuned based on the noise model and the size of the image. For a $512 \times 512$ image corrupted by salt and pepper noise, the choice $\lambda=100 p$ generally works well, where $p$ is given in (1).

Step 5: Replace designated noise pixels by filtering. In SEAA, each pixel $J(i, j)$ designated as noise is replaced by the average of the noise free pixels in a $3 \times 3$ neighborhood about $J(i, j)$. Since the median is 
also an unbiased estimator of location that is known to be robust against heavily tailed noise, it is reasonable to alternatively consider replacing the noise pixels with the median of the noise free pixels in the surrounding neighborhood; this is called Signal Exclusive Adaptive Median filtering (SEAM). Our empirical studies to date suggest that the performances of SEAA and SEAM are similar, with SEAA being consistently better with respect to PSNR when the noise ratio is significant $(\geq 20 \%)$.

\section{Results}

We evaluated and compared the proposed SEAA algorithm against other existing filtering techniques in terms of peak signal-to-noise-ratio (PSNR) defined as

$$
\text { PSNR }=10 \log _{10}\left(\frac{\sum_{n=1}^{N^{2}} 255^{2}}{\sum_{n=1}^{N^{2}}(\hat{p}-p)^{2}}\right)
$$

The competing techniques include the standard $3 \times 3$ median filter, minimum-maximum exclusive mean filter (MMEM) [3], adaptive center-weighted median filter (ACWMF) [4] and conditional signal-adaptive median filter (CSAM) [5].

Denoised image results obtained by applying SEAA to three instances of the $512 \times 512$ gray scale Lena image corrupted by fixed-value impulse noise with various noise ratios are presented in Figure 4. For a $3 \%$ noise ratio, the PSNR of the denoised image is $44.9 \mathrm{~dB}$. A PSNR of $43.3 \mathrm{~dB}$ is obtained when the noise ratio is increased to $5 \%$. With the noise ratio dramatically increased to $40 \%, 50 \%$ or even $60 \%$, we obtain, respectively, a PSNR of $33.71 \mathrm{~dB}, 32.33 \mathrm{~dB}$ and $29.89 \mathrm{~dB}$. It is worth noting that even better results with regard to PSNR can be obtained from SSEA in the presence of high noise ratios if the window size is increased from $3 \times 3$ to $5 \times 5$, although this involves increased computational complexity. The denoising performance of SSEA and SEAM in terms of PSNR relative to competing recent nonlinear techniques is summarized in Table 1, where it can be seen that SSEA offers an attractive tradeoff between noise suppression, detail preservation, and computational complexity.

\section{Differences between SSEA and CSAM}

The homogeneity level concept was first proposed by Pok et al. in [5]. In SSEA, we have further developed and exploited this concept. Here, we highlight several major conceptual differences between SSEA and the CSAM algorithm: a) In CSAM, any two pixels are classified as either homogeneous or nonhomogeneous. In SEAA, we have used the count of the number of zeros in the map $M_{i, j}$ to introduce a quantitative homogeneity measure providing a spectrum of descriptions between the extremes of homogeneous and non-homogeneous. b) SEAA unifies the notion of "connectedness" that is associated with the CSAM algorithm with the traditional notion of connected components labeling. In particular, the notion of connectedness in the CSAM algorithm is not transitive, whereas that associated with SEAA is transitive. c) SEAA eliminates the need for the computationally expensive recursive search process required by CSAM. d) finally, the estimation filter used in SEAA and SEAM considers only the noisefree pixels within the relevant neighborhood, whereas the estimator in the CSAM algorithm involves all the pixels in a $3 \times 3$ or $5 \times 5$ window.

Table 1. PSNR (dB) of denoised images obtained from the impulse noise corrupted Lena image.

\begin{tabular}{c|c|c|c|c|c|c}
\hline \multirow{2}{*}{$\begin{array}{c}\text { Noise } \\
\text { ratios }\end{array}$} & \multicolumn{5}{|c}{ PSNR (dB) results for 512 x 512 Lena image } \\
\begin{tabular}{c|c}
$3 \times 3$ \\
$10 \%$
\end{tabular} & $\begin{array}{c}\text { MMEM } \\
{[3]}\end{array}$ & $\begin{array}{c}\text { ACWMF } \\
{[4]}\end{array}$ & $\begin{array}{c}\text { CSAM } \\
{[5]}\end{array}$ & SEAA & SEAM \\
\hline $20 \%$ & 29.69 & 33.86 & 36.54 & 36.44 & 37.58 & 37.55 \\
\hline $30 \%$ & 27.20 & 32.60 & 33.68 & 34.32 & 35.57 & 35.22 \\
\hline
\end{tabular}

\section{Conclusion}

We presented a novel signal exclusive algorithm for removal of impulse noise. Corrupted pixels were estimated using a linear (SEAA) or nonlinear (SEAM) location estimator applied selectively to only those pixels labeled as noise free. We developed an extended notion of the concept of homogeneity level map and used it to detect noise and noise candidate pixels. We also introduced a new approach for reducing the labels of noise candidate pixels to noise or signal. The results in Table 1 show that SEAA is an attractive alternative in terms of denoising performance traded against computational complexity. The main novelty of this approach lies in the estimation of the true values of noisy image pixels from only the noise free values in a local neighborhood of the observed image. Within this framework, the linear estimator performed best in high noise environments. 


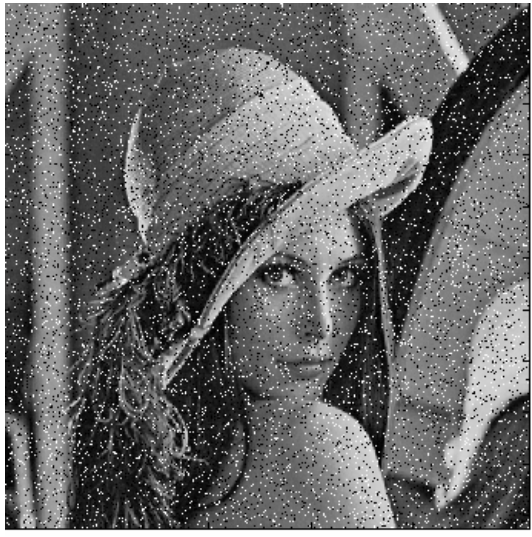

(a)

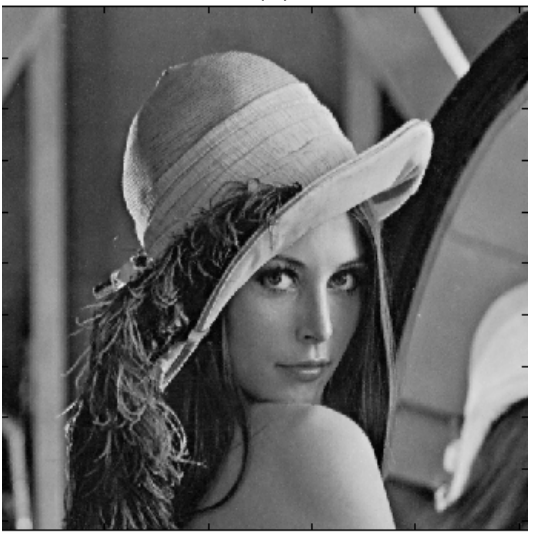

(d)

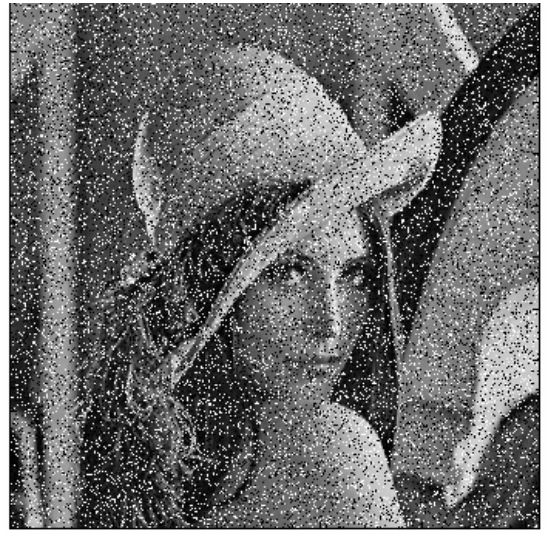

(b)

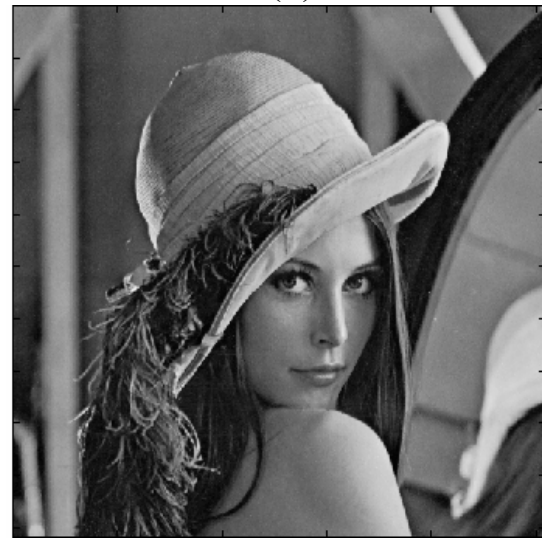

(e)

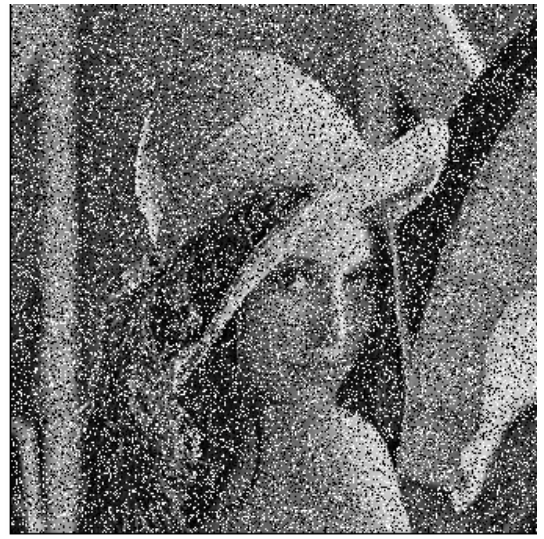

(c)

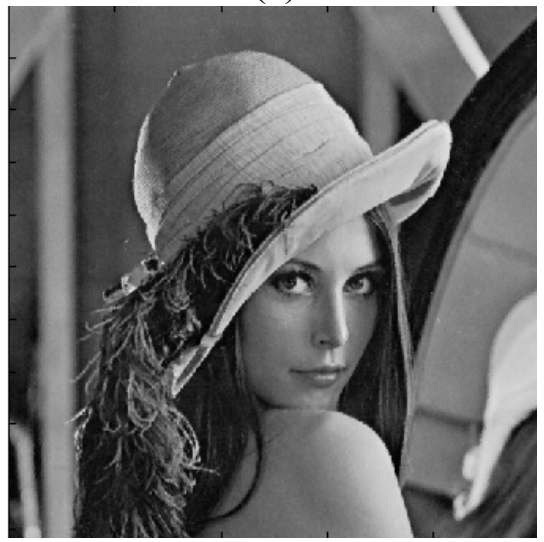

(f)

Figure 4. Simulation results for (a) $10 \%$, (b) $20 \%$, and (c) $30 \%$ impulse noise corrupted "Lena" image, with corresponding restored image results produced by SEAA (d)-(f).

\section{References}

[1] R. C. Gonzalez and R. E. Woods, Digital Image Processing, 2nd ed. Englewood Cliffs, NJ: PrenticeHall, 2001.

[2] A. Bovik, Handbook of Image \& Video Processing, $1^{\text {st }}$ Ed. New York: Academic, 2000

[3] W.-Y. Han and J.-C. Lin, "Minimum-maximum exclusive mean (MMEM) filter to remove impulse noise from highly corrupted images," Electron. Lett., vol. 33, no. 2, pp. 124-125, 1997.

[4] T. Chen and H. R. Wu, "Adaptive impulse detection using center-weighted median filters," IEEE Signal Processing Lett., vol. 8, pp. 1-3, Jan. 2001.

[5] G. Pok, J.-C. Liu, and A. S. Nair, "Selective removal of impulse noise based on homogeneity level information," IEEE Trans. Image Processing, vol. 12, pp. 85-92, Jan. 2003. 